Collection SFN 8 (2007) 161-177

(C) EDP Sciences, Les Ulis

DOI: 10.1051/sfn:2007013

\title{
Systèmes hôtes nanométriques : les nanotubes de carbone
}

\author{
J.-L. Sauvajol, S. Rols, J. Cambedouzou et R. Almairac
}

Laboratoire des Colloïdes, Verres et Nanomatériaux, Université Montpellier II, 34095 Montpellier Cedex 5, France

\section{INTRODUCTION}

Les nanotubes de carbone ont fait l'objet d'un grand nombre d'études depuis leur découverte au début des années 90 par Iijima et collaborateurs [1,2]. Un nanotube mono feuillet est issu de l'enroulement d'un plan de graphite en cylindre [3]. Comme nous le verrons par la suite, ces nanotubes monofeuillets s'auto-organisent en phase solide pour former des faisceaux de nanotubes, et c'est à ce type de matériaux nanostructurés que nous nous intéresserons. On rappellera qu'il existe un deuxième type d'organisation des nanotubes : les nanotubes multifeuillets, qui peuvent être vus comme des molécules cylindriques formées de 2 à 50 nanotubes imbriqués les uns dans les autres à la manirère des poupées russes [1].

Un échantillon macroscopique de nanotubes monofeuillets, préparé par la méthode de l'arc électrique [4] ou par ablation laser [5], présente une structure fibrillaire, les fibres ne présentant aucune orientation préférentielle (Figure 1(a)). Une analyse de ces fibres par microscopie électronique en transmission haute résolution (METHR) montre que les nanotubes s'organisent en faisceaux de tubes parallèles les uns aux autres (Figure 1(b)). Dans chaque faisceau, les diamètres des nanotubes sont voisins. Par contre ils peuvent être très différents d'un faisceau à l'autre. Ceci est à l'origine de la distribution en diamètre qui caractérise en général (sauf pour des conditions de synthèse très particulières) les échantillons massifs de nanotube de carbone monofeuillet. D'autre part la taille de ces faisceaux est elle-même distribuée. Ainsi, le nombre de tubes dans un faisceau peut varier d'une dizaine à une centaine suivant les méthodes de synthèse, ou même d'une zone à l'autre à l'intérieur d'un même échantillon.
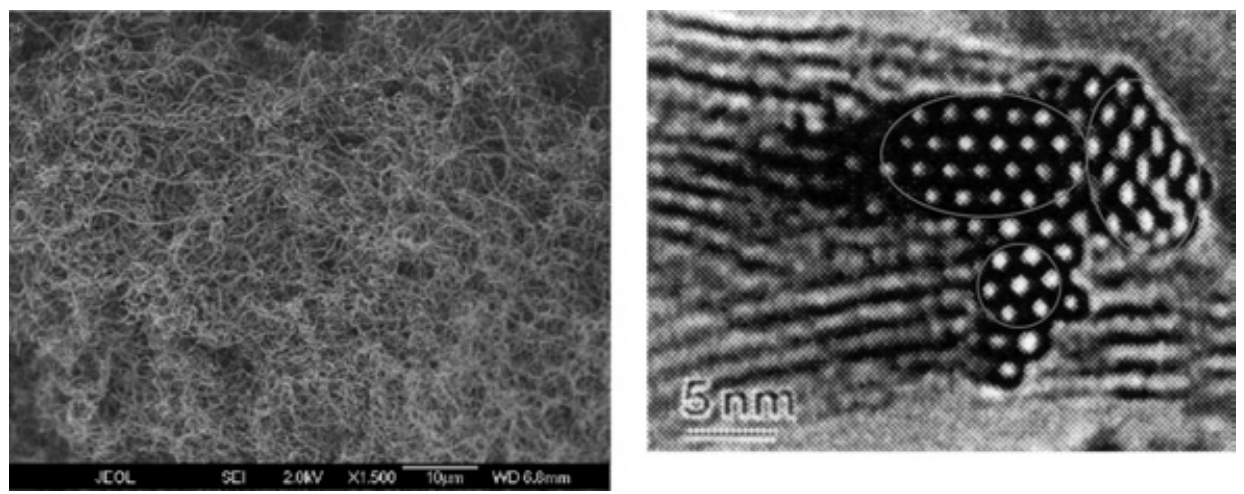

Figure 1. Gauche : microscopie électronique à balayage d'un échantillon macroscopique de nanotubes monofeuillets. Droite : microscopie électronique à transmission haute résolution d'une fibre comportant au moins 3 faisceaux de nanotubes monofeuillets.

Un échantillon macroscopique peut donc être vu comme un ensemble de faisceaux de nanotubes orientés de manière isotrope. En accord avec les clichés de METHR (Figure 1), un faisceau de nanotubes 
sera assimilé à un arrangement de nanotubes parallèles sur un réseau hexagonal compact $2 D$ de taille finie. Schématiquement, l'organisation «idéale » (la plus symétrique) des nanotubes en faisceau est celle représentée sur la Figure 2. Le paramètre de ce réseau est défini comme la somme du diamètre des tubes et de la distance entre tubes (Figure 2). Cette distance est voisine de celle que l'on trouve entre deux plans dans le graphite, soit $0.34 \mathrm{~nm}$. Comme le problème qui nous intéressera par la suite est celui de l'insertion d'atomes ou de molécules dans les faisceaux, ce schéma permet également de définir les quatre sites d'insertion/adsorption les plus probables. A la surface des faisceaux, on distingue deux types de sites : la surface des tubes $(\mathrm{S})$ et les rainures entre deux tubes $(\mathrm{G})$. A l'intérieur des faisceaux on a deux autres sites : les interstices entre trois tubes (I) et l'intérieur des tubes (T) à la condition que ces derniers soient ouverts. Notons que si on nomme $j$ le nombre de tubes suivant une facette du faisceau les nombres de sites $\mathrm{G}$, I et T sont respectivement : $6(j-1), 6[j(j-2)+1]$ et $1+3 j(j-1)$.

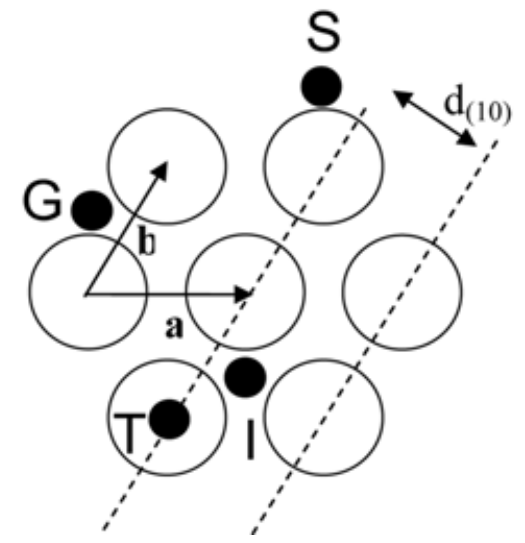

Figure 2. Image schématique de la représentation d'un faisceau de nanotubes. Les sites d'insertion les plus probables sont indiqués par des ronds pleins. Les paramètres du réseau $2 \mathrm{D}$ sont représentés par les vecteurs de la maille unitaire $a$ et $b$. Deux plans de type $(1,0)$ sont indiqués ainsi que leur distance inter réticulaire.

Les techniques de diffraction $X$ et neutrons permettent de déduire les principales caractéristiques de ces faisceaux en terme de diamètre moyen des tubes, de largeur de la distribution en diamètre et de taille moyenne des faisceaux. La longueur des tubes joue également un rôle dans le profil du diffractogramme. Si l'on ne s'intéresse qu'aux caractéristiques des faisceaux-c'est-à-dire si l'on se limite à l'information du diagramme de diffraction pour des valeurs du vecteur de diffusion inférieures à $\sim 2.5 \AA^{-1}-$ la structure atomique des tubes peut être oubliée. Les nanotubes sont alors assimilés à des tubes infinis, creux, et présentant en diffraction neutronique une densité de longueur de diffusion nucléaire surfacique homogène. Le facteur de forme d'un tel objet est une fonction de Bessel cylindrique d'ordre $0[4,5,6]$.

Notre but est de donner les outils nécessaires à l'interprétation des diagrammes de diffraction de nanotubes de carbones insérés par des atomes ou des molécules et d'en déduire alors une information sur leur localisation. Avant d'aller plus loin, nous allons rappeler l'interprétation des diagrammes de diffraction d'un ensemble de faisceaux de nanotubes monofeuillets.

\section{MODÉLISATION DES DIAGRAMMES DE DIFFRACTION DES FAISCEAUX DE NANOTUBES}

Un exemple de diffractogramme typique d'un échantillon macroscopique de nanotubes monofeuillets est montré sur la Figure 3. Il est caractérisé par la présence de pics de diffraction aux petites valeurs du 
vecteur de diffusion $Q$. Ces pics annotés (hk) sont attribués aux réflexions sur les plans réticulaires du réseau hexagonal 2- $D$ des tubes en faisceaux (voir la Figure 2). Le pic dominant autour de $0.4 \AA^{-1}$ est associé à la réflexion (10). Comme nous le verrons par la suite, ce pic de diffraction est une sonde très sensible aux changements structuraux induits par l'insertion.

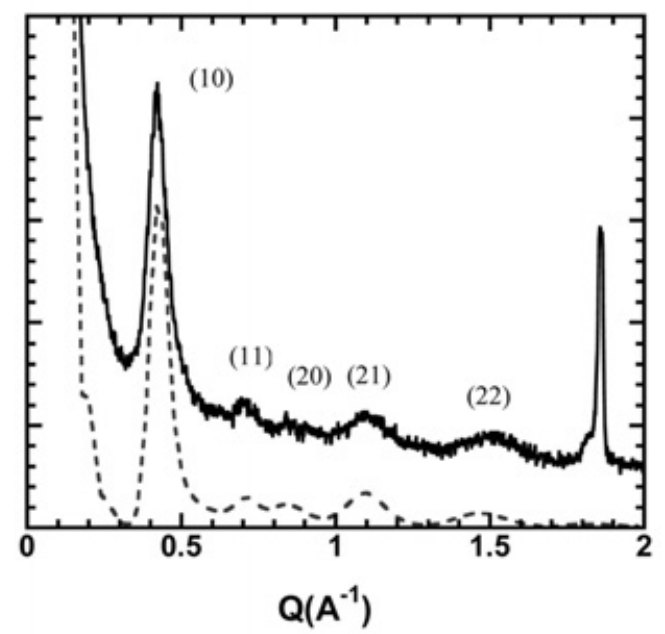

Figure 3. Exemple de diffractogramme mesuré par diffraction neutronique d'un échantillon macroscopique de nanotubes monofeuillets. Les indices des différents pics de diffraction correspondant à des réflexions sur des plans du réseau $2 D$ sont indiqués. Le pic fin non indexé est dû aux résidus graphitiques présents dans l'échantillon. La courbe en pointillé est un ajustement de ce diffractogramme. Les courbes sont décalées pour plus de visibilité.

A cause de la faible cristallinité de ces matériaux ainsi que ses dimensions nanométriques et de part son anisotropie, il est très dangereux de faire une interprétation «cristallographique » classique de ce diagramme. En particulier, il a été démontré que la position du maximum des pics n'était plus directement reliée à la valeur moyenne du paramètre de maille [6,7]. La simplicité de ce diagramme n'est donc qu'apparente et il est indispensable de développer un modèle numérique capable de prendre en compte au mieux la nature des échantillons pour tirer une information quantitative fiable sur le paramètre moyen du réseau (et donc du diamètre moyen des tubes), sur la largeur de la distribution en diamètre et sur la taille des faisceaux.

La procédure que nous avons adoptée est la suivante : on calcule le diagramme de diffraction d'un faisceau comportant un nombre fini de tubes de longueur $\mathrm{L}$ et de même diamètre, dont la direction principale (celle de l'axe des tubes) est distribuée de manière isotrope dans l'espace (moyenne de poudre). Dans ce cas, la moyenne de poudre peut s'écrire :

$$
I\left(D_{0}, Q\right) \propto \frac{1}{4 \pi Q^{2}} \iint\left|A_{T}\left(D_{0}, \vec{Q}\right)\right|^{2} d^{2} S_{Q}
$$

Avec $A_{T}\left(D_{0}, \vec{Q}\right)$, l'amplitude de diffusion définie comme la transformée de Fourier spatiale de la densité de longueur de diffusion cohérente des tubes dans le cas de la diffraction neutronique (densité de charge dans le cas de la diffraction des rayons $\mathrm{X}$ ). 
On peut montrer que cette intensité diffractée s'écrit [6] :

$$
I\left(D_{0}, Q\right)=\frac{I_{0}}{2 N^{2}} \int_{0}^{\pi} \sin \theta\left[\frac{\sin (Q \cos \theta L / 2)}{(Q \cos \theta L / 2)}\right]^{2}\left[J_{0}\left(Q \sin \theta \frac{D_{0}}{2}\right)\right]^{2} \sum_{i j}^{N} J_{0}\left(Q \sin \theta R_{i j}\right) d \theta
$$

où $\theta$ est l'angle entre la direction du vecteur $Q$ et l'axe d'élongation du faisceau, L la longueur des tubes, $N$ le nombre de tubes dans le faisceau, $D_{0}$ le diamètre des tubes, $\vec{R}_{i j}=\vec{R}_{i}-\vec{R}_{j}$ le vecteur du réseau $2 D$ joignant les centres des tubes $i$ et $j$, et $I_{0}$, l'intensité en $\mathrm{Q}=0$, qui s'exprime par :

$$
I_{0}=\left(N S \widetilde{b}_{C}\right)^{2}
$$

où $\mathrm{S}$ est la surface du tube et $\widetilde{b}_{C}$ la longueur de diffusion surfacique qui dans la cas d'un nanotube est égale à :

$$
\widetilde{b}_{C}=\frac{4 b_{C}}{3 \sqrt{3} a_{C-C}^{2}}
$$

$b_{C}$ étant la longueur de diffusion cohérente du carbone et $a_{C-C}$ la distance carbone-carbone à la surface du tube.

Dans un deuxième temps on fait la moyenne sur la distribution en diamètre des tubes.

$$
I(Q)=\frac{1}{2 \sigma_{D} \sqrt{\pi}} \int_{0}^{\infty} I\left(D_{0}, Q\right) \exp \left[-\left(\frac{D-D_{0}}{2 \sigma_{D}}\right)^{2}\right] d D
$$

Sur cette base on fait varier les différents paramètres du modèle afin d'ajuster au mieux le diffractogramme expérimental et de rendre compte des positions, largeurs et intensités relatives des différents pics. Ce type de calcul permet de comprendre en particulier les modulations du diffractogramme qui sont liées à une balance assez subtile entre les effets du facteur de forme des tubes et la position des raies. Ces deux paramètres dépendent directement du diamètre moyen des tubes, de la largeur de sa distribution et du paramètre de Van der Waals. Un type d'ajustement est montré sur la Figure 3 (ligne pointillée). Les positions, largeurs et intensités relatives des différents pics de Bragg sont restituées de manière satisfaisante. De cet ajustement on dérive que le paramètre moyen du réseau 2D de l'échantillon considéré est $1.73 \mathrm{~nm}$ et donc que le diamètre moyen des tubes est de $1.40 \mathrm{~nm}$, la largeur de la distribution est de $0.2 \mathrm{~nm}$ et le nombre de tubes dans les faisceaux de l'ordre de 20 .

\section{INSERTION D'ATOMES OU DE MOLÉCULES DANS DES FAISCEAUX DE NANOTUBES DE CARBONE : L'APPORT DE LA DIFFRACTION NEUTRONIQUE Ä LA DÉTERMINATION DES DIFFÉRENTS SITES D'INSERTION}

Sur la base de calculs du même type que ceux montrés précédemment on peut prédire les changements les plus significatifs liés à la présence de matière diffusante en différents sites du réseau. Nous nous focalisons par la suite sur l'évolution du pic (10). En reprenant la notation du paragraphe précédent, on peut écrire l'intensité diffusée par un système binaire composé de tubes de carbone en faisceau et d'atomes (ou molécules) insérés sous la forme :

$$
I(Q) \propto \frac{1}{4 \pi Q^{2}} \iint\left|A_{T}(\vec{Q})+A_{I}(\vec{Q})\right|^{2} d^{2} S_{Q}=I^{T}(Q)+I^{I}(Q)+T C(Q)
$$

où $A_{T}(Q)$ est l'amplitude diffusée par les tubes et $A_{I}(Q)$ celle diffusée par l'espèce (atome ou molécule) insérée. Bien entendu, nous avons $A_{T}(Q) \propto b_{c}$ et $A_{I}(Q) \propto b_{I}$ où $b_{c}$ et $b_{I}$ sont les longueurs de diffusion cohérente du carbone et de l'espèce insérée respectivement. Il est possible de calculer relativement facilement les amplitudes de diffusion en faisant des hypothèses simples sur la répartition des molécules 
insérées dans les faisceaux : on prendra une répartition surfacique représentée par une densité de surface de longueur de diffusion quand une monocouche moléculaire ou atomique est formée ; on prendra une répartition volumique représentée par une densité volumique de longueur de diffusion quand les molécules adsorbées forment des amas et/ou quand leur position est distribuée dans un certain volume.

En développant le carré, on fait apparaître un terme purement lié au réseau de nanotube $\left(I^{T}(Q) \propto b_{c}^{2}\right)$, un terme purement lié aux molécules insérées $\left(I^{I}(Q) \propto b_{I}^{2}\right)$ ainsi qu'un terme qui traduit les corrélations entre les tubes et les atomes (molécules) insérés et que nous désignerons par « terme d'interférences croisées » $\left(T C(Q) \propto b_{c} b_{I}\right)$. Un des avantages de la diffusion neutronique est de pouvoir jouer sur le poids respectif de ces composantes par le biais de la substitution isotopique. Nous allons voir 2 exemples qui tirent ainsi profit de la possibilité de variation de contraste. Mais auparavant, voyons quel est l'effet de l'insertion dans les faisceaux de nanotubes au niveau des résultats numériques.

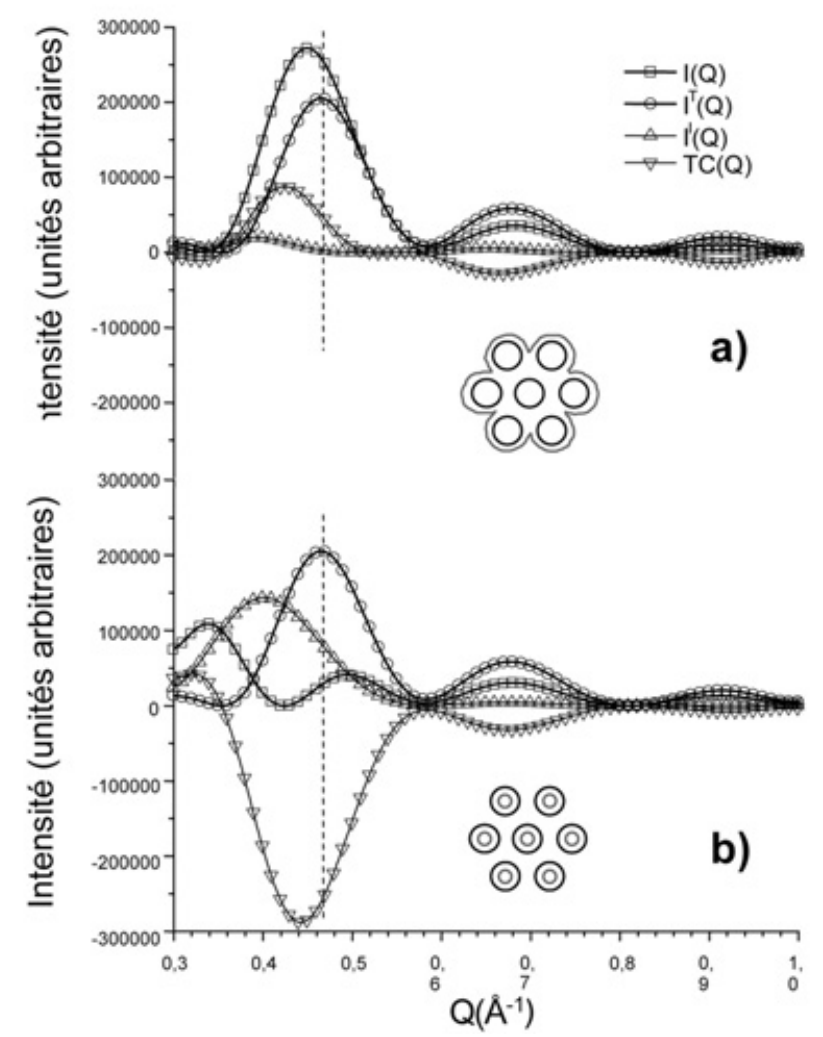

Figure 4. Diagrammes théoriques de diffraction pour 2 modèles d'adsorption différents : (a) les molécules adsorbées forment une monocouche à l'extérieur des faisceaux, (b) les molécules adsorbées forment une monocouche à la surface intérieure des nanotubes.

Pour ces deux modèles, les contributions $I^{T}(Q), I^{I}(Q)$ et $T C(Q)$ à $I(Q)$ sont montrées. On notera que le diagramme de diffraction du faisceau sans adsorbant est $I^{T}(Q)$ et que la structure du faisceau reste inchangée par l'adsorption.

La Figure 4 présente le diagramme de diffraction calculé pour 2 modèles d'insertion différents de $l^{\prime} \mathrm{Ar}^{36}$ dans un faisceau de nanotubes de carbone composé de 7 tubes de diamètre $13.6 \AA$ et d'une 
longueur de $1000 \AA$ A. Nous n'avons pas tenu compte d'une quelconque distribution en diamètre dans cet exemple. La distance minimum entre 2 tubes adjacents a été choisie à 3.2 A. Dans le modèle a) on fait l'hypothèse du recouvrement de la surface extérieure des faisceaux par une monocouche d'Ar de densité uniforme. Dans le modèle b) cette monocouche vient tapisser la paroi intérieure des nanotubes. Les différentes courbes sont relatives aux termes que nous avons explicités dans l'équation 3. En particulier nous pouvons comparer les diagrammes $I(Q)$ et $I^{T}(Q)$ qui représentent l'intensité diffractée après et avant adsorption respectivement. On remarque que l'effet majeur pour le modèle a) où la surface extérieure des faisceaux est peuplée, est une augmentation de l'intensité de la raie (10) ainsi qu'un déplacement de celle-ci vers les faibles valeurs de $Q$. Au contraire, le modèle b) indique que la présence de molécules à l'intérieur des tubes fait chuter de façon très importante l'intensité de la raie (10). Ces deux effets contraires sont dus en particulier au terme d'interférences croisées $T C(Q)$ qui peut être négatif et qui est le principal responsable de la chute d'intensité de la raie (10) dans le modèle b).

Il est aussi possible de considérer une distribution volumique de diffuseur. Ceci peut être nécessaire dans le cas ou la molécule adsorbée possède une structure interne complexe et occupe un volume important de l'espace, ou quand l'on s'approche de la condensation capillaire avec des gaz. Il est aussi nécessaire de considérer une distribution volumique quand la position des atomes est distribuée dans un certain volume (diffusion dans un espace confiné par exemple) et fluctuante dans le temps.

En reprenant les hypothèses du calcul indiquées précédemment, on peut écrire l'intensité diffusée par des faisceaux de nanotubes dans lesquels de la matière est insérée dans les tubes, celle-ci étant assimilée

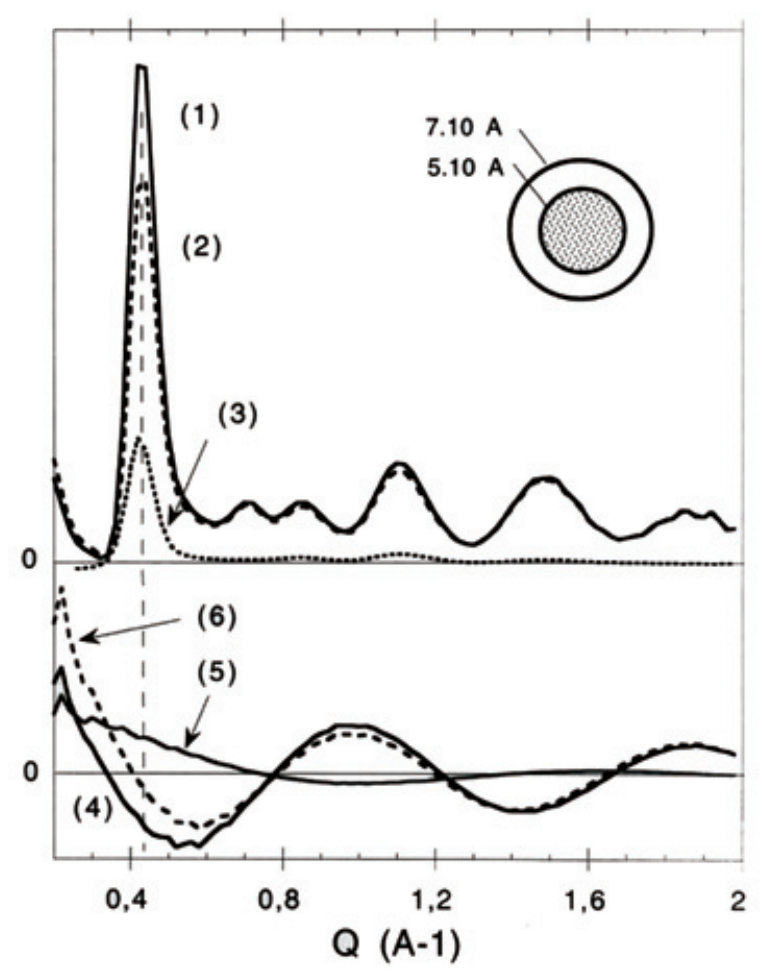

Figure 5. Evolution du pic (10) : courbe 1, faisceaux de tubes vides ; courbe 2, faisceaux de tube pleins, courbe 3 , différence 1-2. Amplitude des facteurs de forme : courbe 4, tube ; courbe 5 gaz, courbe 6 , tube + gaz. 
à un cylindre plein de diamètre $D_{I}$. Après quelques calculs simples on trouve que cette intensité s'exprime comme :

$$
I\left(D_{0}, Q\right) \approx \int_{0}^{\pi} \sin \theta \sum_{i j}^{N} J_{0}\left(Q \sin \theta R_{i j}\right)\left[b_{C} J_{0}\left(Q \sin \theta \frac{D_{0}}{2}\right)+2 X b_{I} \frac{B\left(Q \sin \theta \frac{D_{I}}{2}\right)}{\left(Q \sin \theta \frac{D_{I}}{2}\right)^{2}}\right]^{2} d \theta
$$

la fonction $B(x)$ est donnée par : $B(x)=\int_{0}^{x} t J_{0}(t) d t$

où $X$ est le taux d'insertion (en \%). La Figure 5 donne comme exemple l'évolution du diagramme pour un taux d'insertion de $2.5 \%$ (dans ce calcul représenté ici, l'espèce insérée est l'oxygène).

Le modèle prédit aussi une chute de l'intensité du pic (10) sans déplacement de celui-ci, comme obtenu avec le modèle b) précédent.

Nous allons maintenant confronter ces prédictions théoriques à des données expérimentales.

\subsection{Exemple 1 : insertion d'Argon dans des faisceaux de nanotubes}

L'étude par diffraction des neutrons de l'insertion d'argon dans des faisceaux de nanotubes offre une bonne illustration de la pertinence de la diffraction neutronique couplée à une simulation du diffractogramme dans la localisation des sites hôtes du faisceau. Les résultats, rapportés ci-dessous, sont empruntés aux études réalisées par M. Bienfait et collaborateurs et Rols et collaborateurs [8,9].

Les expériences ont été réalisées sur des échantillons macroscopiques de faisceaux de nanotubes monofeuillets (diamètre moyen $1.4 \mathrm{~nm}$, largeur de la distribution $0.2 \mathrm{~nm}$ ) dans lesquels a été inséré soit de l'argon $36\left(\mathrm{Ar}^{36}\right)$ dont la section de diffusion cohérente est de 77.9 barns, soit de l'argon $40\left(\mathrm{Ar}^{40}\right)$ dont la section de diffusion cohérente est significativement plus faible, 0.421 barn [8].

La Figure 6 (gauche) montre la courbe de chaleur isostérique en fonction du taux d'insertion. Il est à signaler que dans cette premiére expérience les tubes étaient majoritairement fermés. On observe deux plateaux. Chaque plateau est attribué à des sites ayant des énergies de liaisons voisines. Les calculs des énergies de liaison permettent d'attribuer celui de plus haute énergie ( $3.6 \mathrm{kcal} / \mathrm{mole})$ à l'insertion dans les rainures (sites $\mathrm{G}$ de la Figure 2) et dans les interstices les plus larges situés aux défauts de structure des faisceaux, et celui de plus basse énergie $(2.4 \mathrm{kcal} / \mathrm{mole})$ à l'adsorption à la surface des faisceaux $[8,9,10]$ (les sites $T$ à l'intérieur des tubes n'ont pas à être considérés car les tubes étaient fermés).

Sur la Figure 6 (droite, haut) est représentée l'évolution du pic (10) des faisceaux de nanotubes pour deux taux d'insertion correspondants aux deux plateaux précédents. Les symboles ouverts représentent les données obtenues avec l' $\mathrm{Ar}^{40}$ et les symboles fermés celles obtenues avec $\mathrm{l}^{\prime} \mathrm{Ar}^{36}$. On ne constate pas d'évolution significative du pic (10) pour les expériences réalisées avec l' $\mathrm{Ar}^{40}$. Dans ce cas, l'argon est quasi « invisible » en diffraction neutronique et on ne teste de fait que l'organisation en faisceau des tubes. A l'évidence, celle-ci n'évolue pas du fait de l'insertion d'argon. A l'inverse, les expériences réalisées avec l' $\mathrm{Ar}^{36}$ indiquent un très fort déplacement du pic (10) vers les bas $Q$ et un gain d'intensité significatif pour un taux d'adsorption correspondant au second plateau de chaleur. Un raisonnement classique suggèrerait d'attribuer ce déplacement vers les bas $Q$ à une dilatation du réseau des tubes. En fait il n'en est rien car une telle dilatation aurait dû aussi être observée dans les expériences avec $l^{\prime} \mathrm{Ar}^{40}$. Le calcul de l'évolution du pic (10) sous l'effet de l'insertion d' $\mathrm{Ar}^{36}$ dans un faisceau formé de sept tubes $(10,10)$ permet de rendre compte de ces résultats (Figure 6, droite, bas). On constate que le recouvrement de la surface extérieure des faisceaux par les atomes d'argon explique complètement le déplacement et le gain d'intensité du pic (10) observés expérimentalement. Il faut noter que dans cette étude, les calculs ont été réalisés en effectuant dans un premier temps des simulations de type 
Monte Carlo d'adsorption suivi d'une minimisation de la structure obtenue. Dans un troisième temps, les calculs de diffraction on été effectués en tenant compte de la position de chaque atome de d'argon et de carbone, c'est-à-dire en effectuant un calcul de type atomique. Les résultats obtenus sont en tous points comparables à ceux obtenus par la méthode décrite dans le paragraphe précédent pour des valeurs de $Q$ inférieures à $2.5 \AA^{-1}$ (comparer l'évolution de la (10) sur les Figures 4(a) et 6(b)).
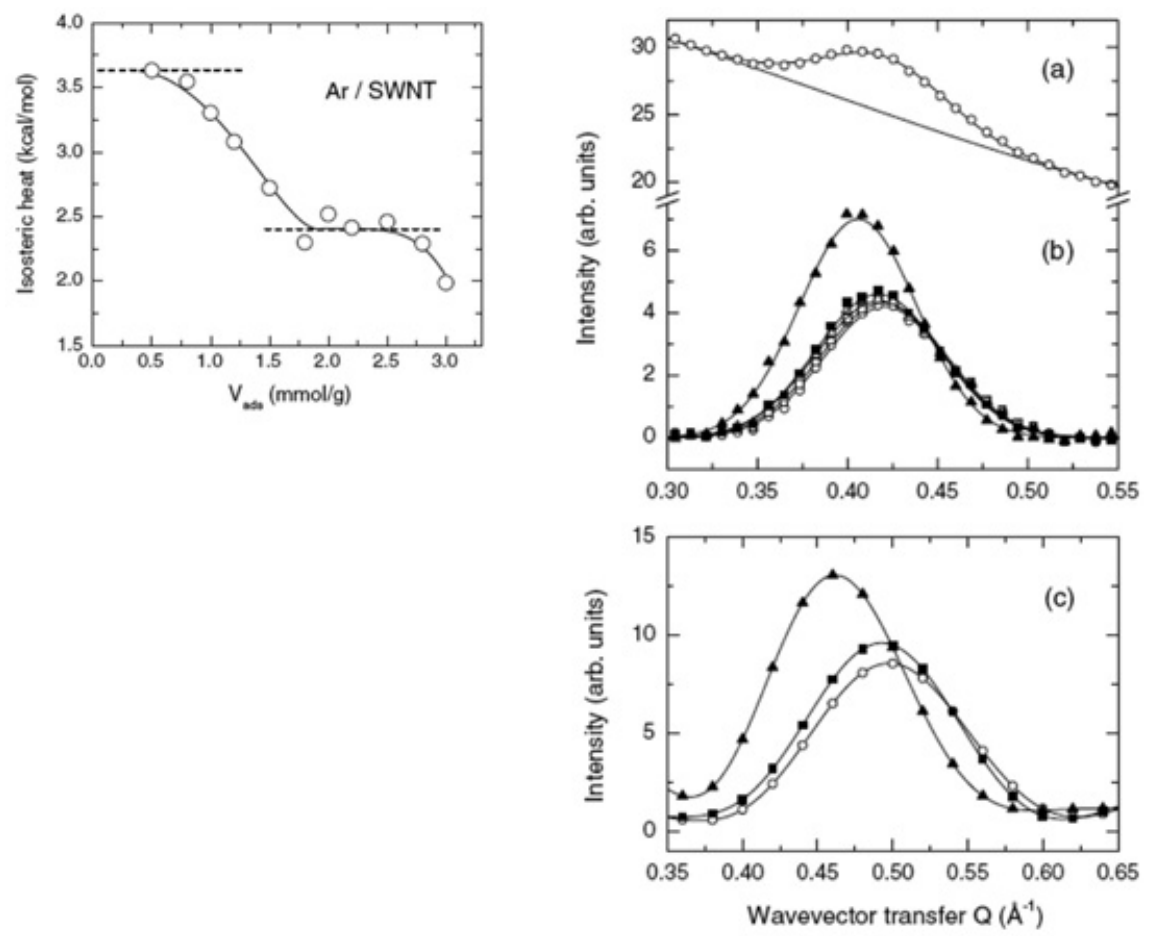

Figure 6. (gauche). Evolution de la chaleur isostérique avec le taux d'insertion, (droite, haut) : (a) diagramme brut avant insertion. (b) Evolution du pic (10) avec le taux d'insertion (le bruit de fond a été retiré). Ronds ouverts : avant insertion, carrés et triangles : pic (10) pour des taux d'insertion correspondant au premier et deuxième plateau de la courbe de chaleur isostérique (symboles ouverts ; insertion avec $\mathrm{l}^{\prime} \mathrm{Ar}^{40}$, symboles pleins : insertion avec $\mathrm{l}^{\prime} \mathrm{Ar}^{36}$. (Droite, bas) : simulation de l'évolution du pic (10) pour un faisceau de sept tubes $(10,10)$ inséré par de $l^{\prime} \mathrm{Ar}^{36}$. Ronds ouverts : pic (10) du faisceau avant insertion ; les carrés et triangles pleins : pic (10) du faisceau après insertion, les taux d'insertion correspondent au premier et deuxième plateau de la courbe de chaleur isostérique respectivement.

Par la suite, une deuxième expérience a été réalisée par Rols et collaborateurs [9] sur des faisceaux de tubes purifiés par attaque chimique. La Figure 7 résume les principaux résultats. D'une part la courbe de chaleur isostérique est la même que pour les tubes fermés à l'exception du premier palier qui est plus étendu et mieux défini. Ceci permet de conclure qu'un nouveau site d'adsorption est rendu accessible par le traitement acide. De plus, ces sites préférentiels d'insertion possèdent une énergie de liaison voisine de celle des rainures. Le résultat le plus remarquable en diffraction neutronique in situ est la chute de l'intensité du pic (10) observée au faible taux d'insertion, et non observée dans l'expérience avec les tubes majoritairement fermés. Cette observation indique que le traitement acide subi par l'échantillon a 
pour effet «d'ouvrir » les nanotubes et ainsi de rendre accessible leur espace intérieur aux atomes d'Ar. Des calculs d'énergie de liaison ont montré que ce site est un site très attractif, au même titre que les rainures.

Ainsi, en accord avec les calculs d'énergie de liaison, la chute d'intensité du pic (10) dès le début du dopage confirme l'insertion préférentielle de l'argon à l'intérieur des tubes quand ces derniers sont ouverts.
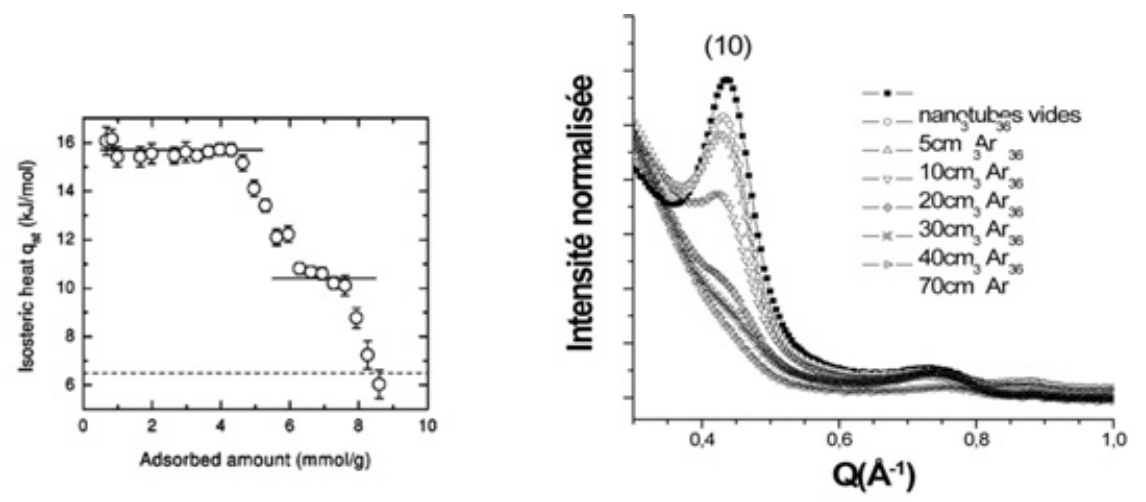

Figure 7. Gauche : Courbe de chaleur isostérique de faisceaux de nanotubes ouverts en fonction du taux d'insertion de l' $\mathrm{Ar}^{36}$. Droite : evolution du pic (10) en fonction du volume d' $\mathrm{Ar}^{36}$ adsorbé.

\subsection{Exemple 2 : les composés ternaires Li-THF-C}

Nous désignons sous l'appellation composé ternaire, un composé où 2 constituants différents sont ajoutés aux nanotubes de carbone monofeuillets, ce qui porte le total des espèces chimiques présentes dans ces échantillons à 3 . Les composés ternaires sont des nanotubes de carbone dans lesquels on a tenté d'insérer des ions alcalins en utilisant une méthode de synthèse dite de « chimie douce » dont l'avantage majeur est le contrôle de la stœchiométrie alcalin/carbone $[11,12,13]$. Toutefois, on s'est très vite rendu compte que des molécules de tétrahydrofurane $\left(\mathrm{C}_{4} \mathrm{H}_{8} \mathrm{O}\right.$, THF), constituant le solvant durant le dopage se retrouvaient dans le produit de la synthèse. Au final, les composés ternaires sont donc constitués par un mélange de nanotubes de carbone monofeuillets, d'ions alcalins $\left(\mathrm{Li}^{+}, \mathrm{K}^{+}, \cdots\right)$ et de THF.

A cause du nombre assez important de constituants dans les composés ternaires, la détermination de la structure de ces objets n'est pas un problème facile. Toutefois, la connaissance des sites d'intercalation des espèces insérées est d'une importance majeure pour prévoir les mécanismes de charge-décharge d'une électrode réalisée avec ce type de matériau. Nous nous sommes donc intéressés à la détermination de la structure de ces échantillons par diffraction des neutrons. En effet, les composés ternaires contiennent une quantité importante d'hydrogène (dans les molécules de THF). Nous verrons que le contraste isotopique entre hydrogène et deutérium propre aux neutrons peut être ici utilisé de manière très efficace afin de résoudre la structure de ces composés.

La Figure 8 compare les diffractogrammes neutrons d'un échantillon de SWNT bruts à ceux d'échantillons de composés ternaires de stœchiométrie $\mathrm{LiC}_{5.88}$ synthétisés en utilisant un solvant à base de THF et de THF deutéré (noté TDF dans la suite).

Si l'on compare le profil du diffractogramme du composé ternaire $\mathrm{LiC}_{5.88}$-THF à celui de la poudre de SWNT bruts, on relève une différence importante dans le domaine des petites valeurs de $Q$. En effet, la raie (10) du réseau triangulaire des tubes dans les faisceaux, située à $Q=0.44 \AA^{-1}$ dans le diagramme de diffraction de l'échantillon de SWNT bruts a disparu dans celui du composé ternaire. On observe 
désormais un minimum à la place de ce pic, entouré par 2 raies relativement larges en 0.3 et $0.6 \AA^{-1}$. Ce profil diffère sensiblement de celui des diagrammes de diffraction usuellement enregistrés sur les échantillons de SWNT insérés. En effet, bien qu'il ait été montré que l'insertion d'alcalins sous phase vapeur conduisait à un léger déplacement de la raie (10) vers les petites valeurs de $Q$, un tel changement dans le profil de diffraction n'a jamais été observé auparavant.

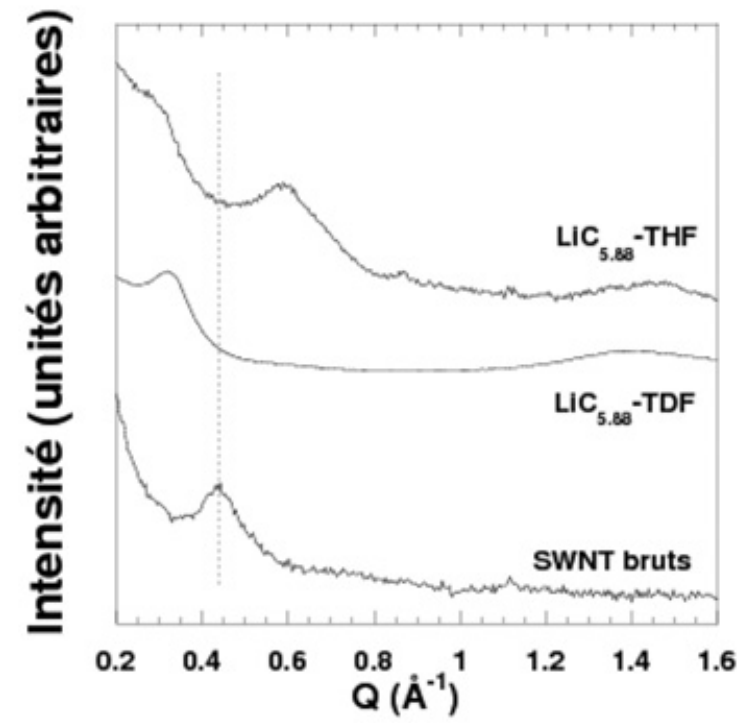

Figure 8. Cliché expérimental de diffraction des neutrons d'un échantillon de composé ternaire de stchiométrie $\mathrm{LiC}_{5.88}$ synthétisé en utilisant un solvant à base de THF (en haut), d'un échantillon deutéré de même stchiométrie (au milieu) et d'un échantillon référence de SWNT bruts (en bas). La ligne en tirets repére la position de la raie (10) des faisceaux dans l'échantillon de SWNT bruts.

Le diagramme de diffraction du composé deutéré $\mathrm{LiC}_{5.88}$-TDF possède un profil encore différent de celui de l'échantillon brut et de celui du composé ternaire hydrogéné. On n'observe plus ici qu'une seule raie de diffraction située à environ $0.3 \AA^{-1}$. La différence de profil entre les diffractogrammes de l'échantillon hydrogéné et de l'échantillon deutéré peut paraître surprenante compte tenu du fait que la deutération des molécules de THF ne doit pas influer sur la chimie de la réaction de synthèse des composés ternaires. Ainsi, la structure du composé ternaire deutéré doit être en toute rigueur identique à celle de l'échantillon hydrogéné.

A ce stade, nous avons eu recours à l'utilisation de la simulation numérique afin de calculer le diagramme de diffraction des composés ternaires en faisant différentes hypothèses sur la localisation des espèces insérées. Nous considèrerons dans un premier temps le diagramme de diffraction du composé ternaire deutéré en faisant l'hypothèse que la raie observée dans le diagramme de diffraction est caractéristique des réflexions sur les plans du réseau triangulaire. On peut ainsi estimer le paramètre de maille de ce réseau à $\sim 24 \AA$ A, ce qui représente une dilatation de $40 \%$. Un travail récent sur la dissolution des composés ternaires [14] nous a permis d'envisager l'hypothèse structurale suivante afin de tenir compte d'une telle dilatation des faisceaux : chaque tube est entouré par une couche d'ions $\mathrm{Li}^{+}$, et de molécules de TDF qui font la jonction entre les tubes décorés. Une image de cette structure est reportée dans la Figure 9.

La Figure 9(a) présente le résultat d'un calcul de dynamique moléculaire d'un composé ternaire dont on a arbitrairement fixé la stIJchiométrie à $\mathrm{LiC}_{6} \mathrm{TDF}_{1.5}$. Le nombre de molécules de TDF était 

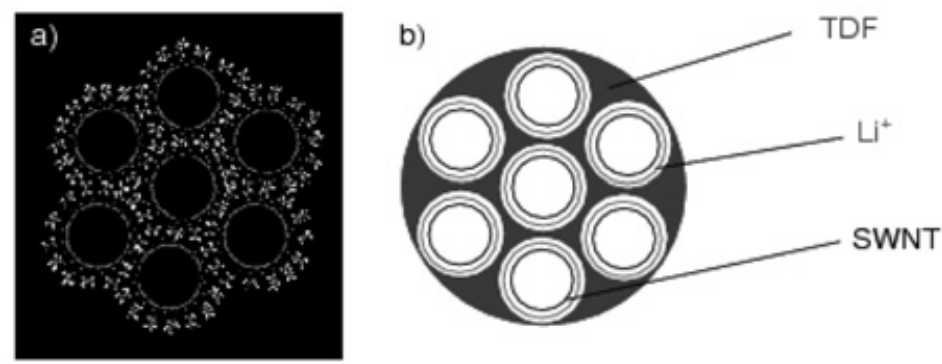

Figure 9. (a) Résultat d'un calcul de minimisation d'énergie d'un composé ternaire de stchiométrie $\operatorname{LiC}_{6} \mathrm{TDF}_{1.5}$, sur la base d'un faisceau de 7 nanotubes. (b) Représentation schématique de la structure du composé ternaire dans l'hypothèse de densités homogènes de diffuseurs.

au départ identique à celui d'ions lithium, mais le calcul a abouti à un rejet de ces molécules hors du faisceau. Le calcul de minimisation d'énergie utilise le champ de forces «Compass» tenant compte des interactions intra-moléculaires et inter-moléculaires (van der Waals et électrostatiques entre les tubes chargés négativement et les ions $\mathrm{Li}^{+}$). Le résultat montre ainsi que la structure obtenue pour $\mathrm{LiC}_{6} \mathrm{TDF}_{1.5}$ est stable. Nous avons utilisé une version simplifiée de cette structure afin de calculer son diagramme de diffraction, comme montré sur la Figure 9(b). Les nanotubes sont remplacés par des cylindres uniformes, tout comme les ions $\mathrm{Li}^{+}$répartis autour des nanotubes. La densité surfacique de ces ions est facilement calculable étant donnés le rayon du cylindre formé par les ions $\mathrm{Li}^{+}$et leur nombre par atome de carbone. Afin de modéliser la réponse due aux molécules de TDF, on considère une densité volumique de ces molécules : on estime le volume occupé par un ion lithium solvaté par 2 molécules de TDF, et on y associe la somme des longueurs de diffusion cohérente de ses constituants. Grâce à ce modèle on peut calculer le diagramme de diffraction de poudre pour les composés ternaires hydrogénés et deutérés, le passage de l'un à l'autre ne nécessitant que la variation de la longueur de diffusion de l'hydrogène.

Après avoir effectué une légère variation des paramètres structuraux issus du calcul, nous avons obtenu un excellent accord entre les diagrammes expérimentaux et modélisés. La Figure 10 illustre cet accord avec un paramètre de maille de $21.3 \AA$, des tubes possédant un diamètre moyen de $13 \AA$ et une distribution gaussienne caractérisée par une largeur totale à mi-hauteur de $3 \AA$ et des faisceaux contenant de 7 à 19 tubes.

Des expériences similaires ont été effectuées sur des nanotubes dopés au potassium en utilisant la même méthode de synthèse. Un composé ternaire de stæchiométrie $\mathrm{KC}_{5.88} \mathrm{THF}$ a ainsi été synthétisé. Un très bon accord entre les diagrammes expérimentaux et calculés a été obtenu en utilisant le modèle précédent mais en effectuant un gonflement de la structure. Le paramètre de maille est ainsi estimé à $23.4 \AA$, le gonflement étant due à la taille plus importante de l'ion $\mathrm{K}^{+}$par rapport à l'ion $\mathrm{Li}^{+}$. Il est très intéressant de constater qu'il est possible de contrôler le paramètre de maille du faisceau -sans en altérer la structure- par le choix de l'atome alcalin $[12,13]$.

Ces deux exemples mettent clairement en évidence l'efficacité de la diffraction neutronique (en jouant sur les effets isotopiques) pour localiser les sites d'absorption d'atomes (ou molécules) à l'intérieur des faisceaux de nanotubes. Il doit être souligné que les données expérimentales doivent être obligatoirement confrontées à des simulations basées sur des hypothèses réalistes afin d'attribuer sans ambiguïté les changements observés en diffraction à l'insertion dans des sites particuliers. Cette méthode a été employée avec succès pour tester l'insertion de toutes sortes de gaz et molécules, avec ou sans transfert de charge [8-10,13,15-17] dans des faisceaux de nanotubes. Elle a été en particulier très efficace pour mettre en évidence l'insertion préférentielle à l'intérieur des tubes d'ion $I_{3}^{-} / I_{5}^{-}$au cours d'un dopage (insertion avec transfert de charge) en phase liquide de faisceaux de nanotubes par de l'iode [17]. 


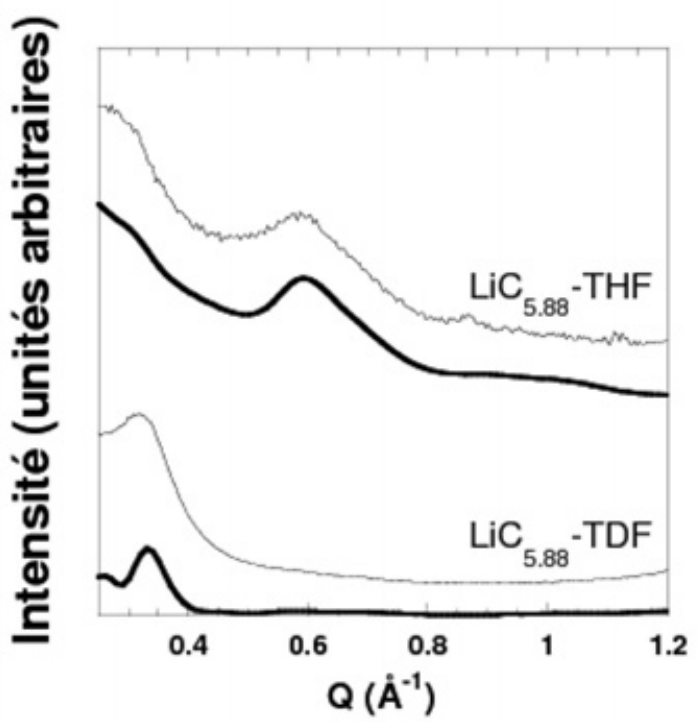

Figure 10. Haut : Diagramme de diffraction expérimental d'un composé ternaire de composition $\mathrm{LiC}_{5.88} \mathrm{THF}$, et calcul du diagramme de diffraction pour un échantillon de $\mathrm{LiC}_{6} \mathrm{THF}$ utilisant le modèle structural décrit dans le texte (trait gras). Bas : Diagramme de diffraction expérimental d'un composé ternaire de composition $\mathrm{LiC}_{5.88} \mathrm{TDF}$, et calcul du diagramme de diffraction pour un échantillon de $\mathrm{LiC}_{6} \mathrm{TDF}$ utilisant le modèle structural décrit dans le texte (trait gras). Une ligne de base de forme $A / Q^{B}$ a été ajoutée aux résultats des simulations pour reproduire le signal aux petites valeurs de $Q$ provenant des impuretés (coques, ...) qui ne sont pas prises en compte dans nos calculs.

\subsection{Exemple 3 : structure des peapods (molécules de $C_{60}$ insérées dans des nanotubes)}

La nanostructure à base de carbone, appelée « peapods », est intéressante à étudier. Cette nanostructure est composée de molécules de $C_{60}$ insérées dans les nanotubes à la manière de pois dans une gousse (d'où l'appellation peapods). L'échantillon macroscopique de peapods utilisé pour cette expérience se présente sous forme de faisceaux, analogues à ceux des nanotubes de départ, et désordonnés en orientation (échantillon de poudre au regard de la diffraction). Les résultats discutés ici ont été obtenus par J. Cambedouzou et collaborateurs $[12,18,19]$

La Figure 11 montre le diffractogramme de cet échantillon (en fait ici ce diffractogramme a été mesuré par diffraction $X$ mais dans le cas d'un matériau constitué d'un seul type d'atome les diffractogrammes $X$ et neutron, en particulier à bas $Q$ sont équivalents). On $y$ retrouve des pics de Bragg reflétant l'organisation en faisceaux (flèches) et d'autres associés à l'organisation des $C_{60}$ à l'intérieur des tubes (flèches à pointe diamant).

Afin d'extraire les informations contenues dans ce diffractogramme nous avons modélisé celui-ci en considérant les molécules de $C_{60}$ comme des sphères présentant une densité surfacique de diffuseur homogène et identique à celle des nanotubes. Un point important de la simulation est d'empêcher toute corrélation de position d'une chaîne de $C_{60}$ d'un tube à l'autre. Pour cela, un décalage aléatoire suivant l'axe du tube, de l'origine de la chaîne de $C_{60}$ pour chacun des tubes a été appliqué. Après quelques manipulations, on arrive à l'expression suivante de l'amplitude diffractée par un faisceau de peapods.

$$
A \approx \sigma_{C} \sum_{h, k=0}^{H, K} \sum_{n_{C}=0}^{N_{C}-1}\left[\iint_{S_{T}} e^{i \vec{Q}\left(n_{C} L \vec{u}_{Z}+\vec{R}(h, k)+\vec{\rho}_{T}\right)} d S_{T}+\iint_{S_{C 60}} e^{i \vec{Q}\left(\left(n_{C} L+x(h, k)\right) \vec{u}_{Z}\right)+\vec{R}(h, k)+\vec{\rho}_{C 60)}} d S_{C 60}\right]
$$



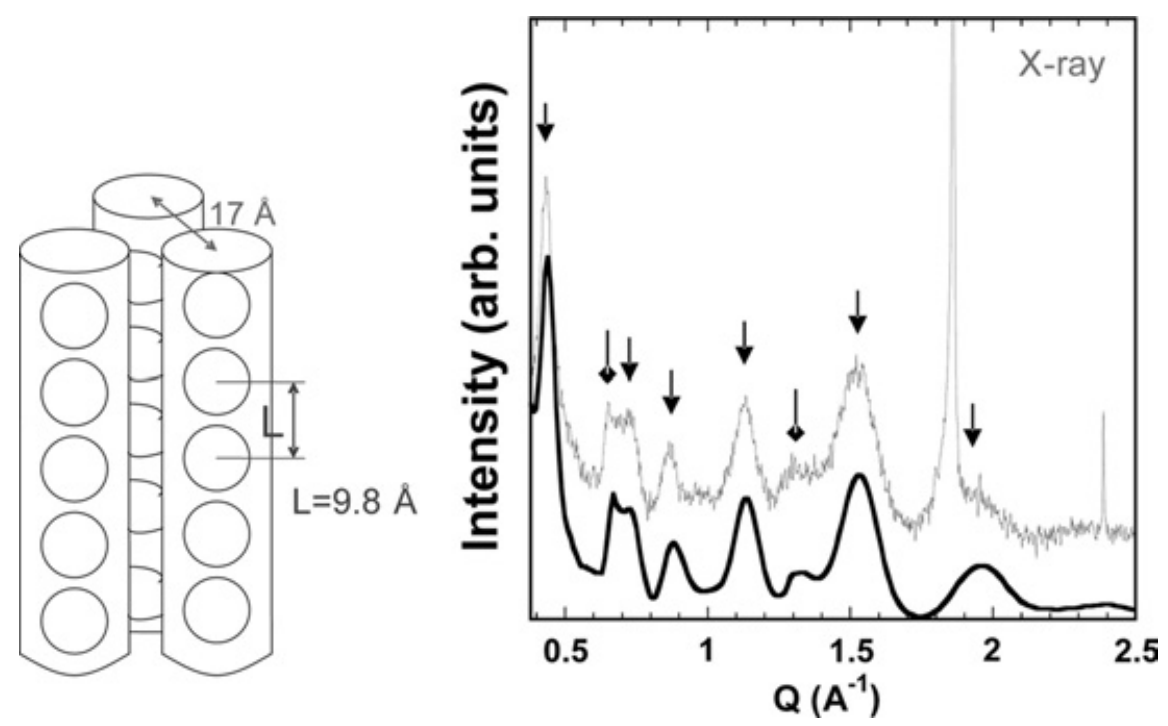

Figure 11. Schéma d'un faisceau de peapods (gauche) et diffractogramme d'un échantillon de peapods (droite). Haut : expérience, bas : simulation.

avec $(H, K)$ valeurs maximum de $(h, k)$ reliées au nombre de tubes, $N_{T}$, dans le faisceau $\left[N_{T}=((H+\right.$ $1)(K+1)] . N_{C}$ est le nombre de cylindres unités, comprenant un $C_{60}$, empilés afin de former un tube. $S_{T}$ et $S_{C 60}$ les surfaces des tubes et des $C_{60}$ respectivement. $x(h, k)$ est la valeur de décalage aléatoire qui permet d'exprimer ce décalage comme : $\vec{T}_{Z}=x(h, k) \vec{u}_{Z}\left(\vec{u}_{Z}\right.$ vecteur unitaire le long de l'axe du tube). Le paramètre $\vec{\rho}_{T}\left(\vec{\rho}_{C 60}\right)$ repère un point du tube (d'un $C_{60}$ ) d'une cellule unité.

Le calcul de l'intensité nécessite de prendre le module carré de l'expression précédente et de faire par la suite une moyenne de poudre pour pouvoir confronter la modélisation au diffractogramme expérimental.

Les calculs des diffractogrammes à l'aide de cette expression permettent d'évaluer l'influence de différents facteurs comme le diamètre des tubes ou le taux de remplissage sur la forme des diffractogrammes. La Figure 12 donne une illustration de cet effet en comparant les simulations pour un même faisceau de tubes vides et de tubes insérés à saturation pas des molécules de $C_{60}$. En plus de l'apparition des pics de Bragg reflétant l'organisation en chaîne des $C_{60}$ dans les tubes (pics centrés à $0.67 \AA$ et $1.35 \AA$ ), on constate la disparition du pic (10) dans les peapods. A sa place on trouve un minimum encadré par deux structures de faibles intensités. Cette évolution est expliquée par la valeur du facteur de forme de l'objet (tubes rempli par des $C_{60}$ ) à la position des pics de Bragg relatifs à l'organisation des tubes en faisceaux. En effet les simulations montrent que la valeur du premier zéro du facteur de forme d'un tube rempli par des $C_{60}$ tombe sur la position du pic (10) des faisceaux correspondant, avec comme conséquence une chute drastique de l'intensité de ce pic. Toutefois, comme la raie est large du fait de la taille finie des faisceaux, les ailes du pic initial restent suffisamment « allumées » par le facteur de forme pour être observables sous la forme de structures faibles de part et d'autre du minimum d'intensité.

Un résultat complet de la modélisation du diffractogramme expérimental est rapporté sur la Figure 11 (courbe en bas, trait en gras). Le calcul reproduit raisonnablement les positions, les largeurs et les intensités relatives des différents pics de Bragg, le meilleur accord expérience/simulation étant obtenu pour une distribution étroite de diamètre autour de $1.35 \mathrm{~nm}$ et un remplissage des tubes de $75 \%$ 


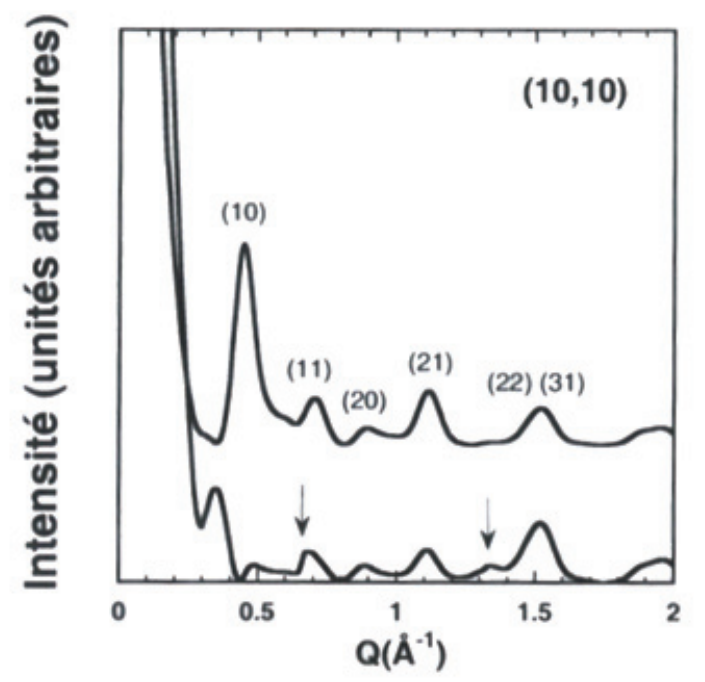

Figure 12. Diffractogrammes calculés pour un faisceau de 12 tubes $(10,10)$ (diamètre $1.36 \mathrm{~nm}$ ) vides (courbe du haut) et insérés à saturation par du $C_{60}$ (courbe du bas). Les flèches indiquent la position des raies relatives à la périodicité des $C_{60}$.

(en accord avec d'autres données connues sur cet échantillon). La distance entre deux molécules de $C_{60}$ est de $9.8 \AA$. On peut insister ici sur le fait que cette distance, plus courte que celle entre $C_{60}$ dans un cristal de $C_{60}$ mais plus longue que celle observée pour des $C_{60}$ pontés par des liaisons de type cyclo-addition $[2+2]$ (comme dans les phases polymères [20]), pose problème. Ce résultat suggère la présence d'oligomères de $C_{60}$ pontés par des liaisons covalentes à l'intérieur des tubes. Les simulations prenant en compte les différentes organisations de $C_{60}$ dans les tubes : monomères, dimères, trimères, peuvent ne montrer aucun changement significatif des diffractogrammes suivant les hypothèses sur la valeur des liaisons covalentes inter- $C_{60}$ et sur la distance séparant 2 molécules non liées [12,22]. Ainsi la question de l'organisation fine des $C_{60}$ insérées dans les tubes ne peut être résolue par le seul outil de la diffraction.

Nous avons montré qu'il pouvait être répondu à cette question à partir de l'étude de la dynamique basse fréquence des molécules de $C_{60}$ dans les tubes. Pour ce type de matériau fortement absorbant, les expériences d'optique (IR et Raman) ne permettent pas de sonder cette dynamique. A l'inverse, la diffusion inélastique des neutrons donne accès à cette information, en permettant l'évaluation de la densité d'états vibrationnelle [21]. Sur la Figure 13 (haut) nous montrons la densité d'états à basse fréquence des $C_{60}$ insérés dans les tubes. Cette densité d'états a été obtenue en soustrayant la densité d'état des faisceaux de nanotubes vides à celle mesurée sur les peapods. On observe l'apparition d'un signal entre 6 et $18 \mathrm{meV}$. On rappellera qu'aucun signal n'est observé dans cette même zone dans la densité d'états mesurée sur les cristaux de $C_{60}$. Les densités d'état vibrationnelles, calculées par la dynamique des réseaux $[12,22]$, indiquent l'apparition de modes additionnels dans cette zone quand on considère des dimères, trimères et polymères de $C_{60}$ insérés dans les tubes (Figure 13).

Ainsi, les données inélastiques de diffusion des neutrons complètent notre information sur la structure des $C_{60}$ à l'intérieur des tubes. Les données inélastiques indiquent clairement la présence de phases oligomères de $C_{60}$ insérées dans les tubes ce qui explique la valeur, obtenue par diffraction, de la distance inter $C_{60}$ qui est une moyenne entre celle mesurée dans un cristal de $C_{60}$ pur et celle mesurée dans les phases polymères du $C_{60}[22]$. 


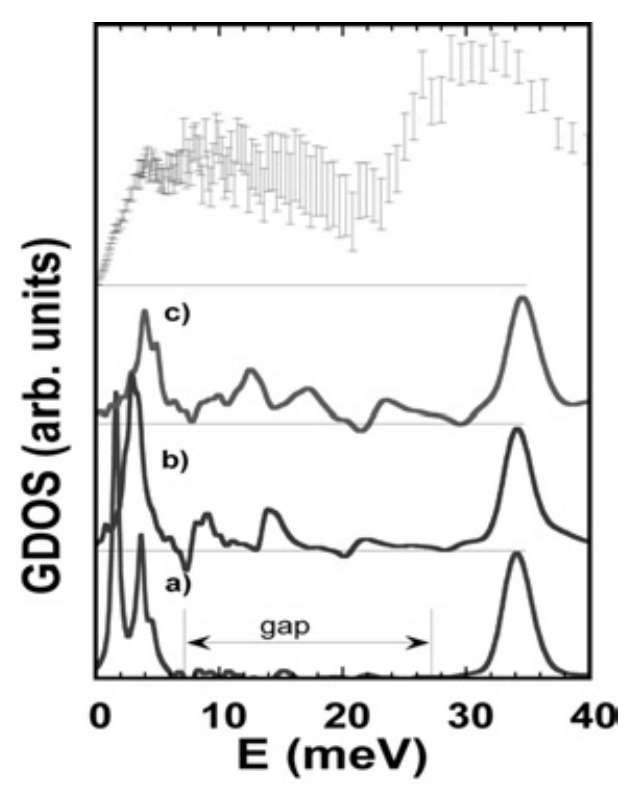

Figure 13. Densité d'états vibrationnelles des $C_{60}$ insérées dans les tubes.

Haut : expérimental.

Bas : Simulations relatives à : (a) une chaîne de monomères de $C_{60}$ insérée dans les tubes, (b) une chaîne de dimères de $C_{60}$ insérée dans les tubes, (c) une chaîne polymérique de $C_{60}$ insérée dans les tubes.

\section{CONCLUSION}

A travers ces quelques exemples, nous avons essayé de montrer comment la diffraction neutronique (et également $X$ ) pouvait donner des informations sur l'organisation des nanotubes en faisceaux d'une part, et sur la localisation d'atomes ou de molécules à l'intérieur des faisceaux d'autre part. On doit toujours insister sur le fait que dégager le maximum d'information de ces données de diffraction implique de développer des simulations numériques qui sont basées sur des hypothèses simples mais suffisantes pour le domaine en $Q$ étudié $\left(Q<2.5 \AA^{-1}\right)$. En particulier dans la mesure où seule l'organisation des tubes en faisceaux, et les changements dans cette organisation induits par l'insertion d'atome ou molécules de gaz, nous intéressent, on a pu considérer les tubes comme des cylindres creux de longueur de diffusion cohérente surfacique homogène et les gaz insérés comme des cylindres pleins de longueur de diffusion cohérente volumique homogène. Sur cette base nous avons pu rendre compte de l'organisation effective de ces matériaux nanostructurés.

Nous avons également montré comment on pouvait obtenir à partir des mesures de diffraction les paramètres moyens (distance entre $C_{60}$, taux de remplissage des tubes) reflétant l'organisation des molécules de $C_{60}$ dans les peapods. L'accord simulation/expérience a validé l'hypoth èse de chaînes de $C_{60}$ non corrélées d'un tube à l'autre. Dans ce cas précis, nous avons pu voir que les mesures de diffraction n'étaient parfois pas suffisantes et qu'elles pouvaient être efficacement complétées par des données de diffusion inélastique des neutrons.

\section{Références}

[1] Iijima, S., Helical Microtubules of Graphitic Carbon, Nature 354, 56, 1991.

[2] Ajayan, P. M. and Iijima, S., Smallest Carbon Nanotube, Nature 358, 23, 1992. 
[3] Pour une revue des principales définitions et propriétés des nanotubes voir, Saito, R., Dresselhaus et M. S., Dresselhaus, G., Physical Properties of Carbon Nanotubes, Imperial College Press, London, 1998.

[4] Journet, C., Maser, W. K., Bernier, P., Loiseau, A., Lamy de la Chapelle, M., Lefrant, S., Deniard, P., Lee, R. and Fischer, J. E., Large-Scale Production of Single-Walled Carbon Nanotubes by the Electric-Arc Technique, Nature, 388, 756, 1997.

[5] Thess, A., Lee, R., Nikolaev, P., Dai, H., Petit, P., Robert, J., Xu, C., Hee Lee, Y., Gon Kim, S., Rinzler, A. G., Colbert, D. T., Scuseria, G. E., Tomanek, D., Fischer, J. E., Smalley, R. E., Crystalline Ropes of Metallic Carbon Nanotubes, Science, 273, 483, 1996.

[6] Rols, S., Almairac, R., Henrard, L., Anglaret, E. and Sauvajol, J.-L., Diffraction by Finite-Size Crystalline Bundles of Single Wall Nanotubes, European Physical Journal B, 10, 263, 1999.

[7] Anglaret, E., Rols S. and Sauvajol, J.-L., Comment on "Effect on the Growth Temperature on the Diameter Distribution and Chirality of Single-Wall Carbon Nanotubes”, Phys. Rev. Lett. 81, 4780,1998 .

[8] Bienfait, M., Zeppenfeld, P., Dupont-Pavlovsky, N., Palmari, J.-P., Johnson, M. R., Wilson, T., DePies, M. and Vilches BienfaitAr, O. E., Adsorption of Argon on Carbon Nanotube Bundles and its Influence on the Bundle Lattice Parameter, Phys. Rev. Lett. 91, 035503, 2003.

[9] Rols, S., Johnson, M. R., Zeppenfeld, P., Bienfait, M., Vilches, O. E. and Schneble, J., Argon Adsorption in Open-Ended Single-Wall Carbon Nanotubes, Phys. Rev. B 71, 155411, 2005.

[10] Johnson, M. R., Rols, S., Wass, P., Muris, M., Bienfait, M., Zeppenfeld, P. Dupont-Pavlovsky, N., Neutron Diffraction and Numerical Modelling Investigation of Methane Adsorption on Bundles of Carbon Nanotubes, Chem. Phys. 293, 217, 2003.

[11] Petit, P., Mathis, C., Journet, C. and Bernier, P., Tuning and Monitoring the Electronic Structure of Carbon Nanotubes, Chemical Physics Letters 305, 370, 1999.

[12] Cambedouzou, J., Structure Etude structurale et vibrationnelle de composés à base de nanotubes de carbone : nanotubes bifeuillets, peapods et composés ternaires, $\mathrm{PhD}$ thesis, Université Montpellier II, 2005.

[13] Cambedouzou, J., Rols, S., Bendiab, N., Almairac, R., Sauvajol, J.-L., Petit, P., Mathis, C., Mirebeau, I., Johnson, M., Tunable Inter-tube Spacing in Single Walled Carbon Nanotube Bundles, Physical Review B 72, R041404, 2005.

[14] Pénicaud, A., Poulin, Ph., Derré, A., Anglaret, E. and Petit, P., Spontaneous dissolution of a singlewalled carbon nanotube salt, Journal of the American Chemical Society, 127, 8, 2004.

[15] Kolesnikov, A. I., Zanotti, J.-M., Loong, C.-K., Thiyagarajan, P., Moravsky, A. P., Loufty, R. O. and Burnham, C. J., Anomalously Soft Dynamics of Water in a Nanotube : A Revelation of Nanoscale Confinement, Phys. Rev. Lett. 93, 035503, 2004.

[16] Bienfait, M., Zeppenfeld, P., Dupont-Pavlovsky, N., Muris, M., Johnson, M. R., Wilson, T., DePies, M. and Vilches, O. E., Thermodynamics and Structure of Hydrogen, Methane, Argon, Oxygen, and Carbon Dioxide Adsorbed on Single-Wall Carbon Nanotube Bundles, Phys. Rev. B 70, 035410, 2004.

[17] Bendiab, N., Almairac, R., Rols, S., Aznar, R., Mirebeau, I. and Sauvajol, J.-L., Structural Determination of Iodine Localization in Single-Walled Carbon Nanotube Bundles by Diffraction Methods, Phys. Rev. B 69, 195415, 2004.

[18] Cambedouzou, J., Pichot, V., Rols, S., Launois, P., Petit, P., Klement, R., Kataura, H. and Almairac, R., On the Diffraction Pattern of $C_{60}$ peapods, European Physical Journal B 42, 31, 2004.

[19] Almairac, R., Cambedouzou, J., Rols, S., Sauvajol, J.-L., Diffraction of Oriented Nanopeapods, European Physical Journal B 49, 147, 2006. 
[20] Davydov, V.A., Kashevarova, L.S., Rakhmanina, A.V., Senyavin, V.F., Càolin, R., Swarc, H., Allouchi, H. and Agafonov, V., Spectroscopic Study of Pressure-Polymerized Phases of $C_{60}$, Physical Review B 61, 11936, 2000.

[21] Bée, M., Quasielastic neutron scattering : Principles and Applications in Solid State Chemistry, Biology and Material Science, Adam Hilger, Bristol, 1988.

[22] Cambedouzou, J., Rols, S., Almairac, R., Sauvajol, J.-L., Kataura, H. and Schober, H., Low-Frequency Excitations of $C_{60}$ Chains Inserted Inside Single-Walled Carbon Nanotubes, Physical Review B 71, R041403, 2005. 